\title{
Large, Uni-directional Actuation in Dielectric Elastomers Achieved By Fiber Stiffening
}

\section{Citation}

Huang, Jiangshui, Tongqing Lu, Jian Zhu, David R. Clarke, and Zhigang Suo. 2012. Large, unidirectional actuation in dielectric elastomers achieved by fiber stiffening. Applied Physics Letters 100(21): 211901.

\section{Published Version}

doi:10.1063/1.4720181

\section{Permanent link}

http://nrs.harvard.edu/urn-3:HUL.InstRepos:9639966

\section{Terms of Use}

This article was downloaded from Harvard University's DASH repository, and is made available under the terms and conditions applicable to Open Access Policy Articles, as set forth at http:// nrs.harvard.edu/urn-3:HUL.InstRepos:dash.current.terms-of-use\#OAP

\section{Share Your Story}

The Harvard community has made this article openly available.

Please share how this access benefits you. Submit a story.

Accessibility 


\title{
LARGE, UNI-DIRECTIONAL ACTUATION IN DIELECTRIC ELASTOMERS ACHIEVED BY FIBER STIFFENING
}

Jiangshui Huang1, Tongqing Lư ${ }^{1,2}$, Jian Zhu' ${ }^{1}$, David R. Clarke,a, Zhigang Suo ${ }^{1, b}$

${ }^{1}$ School of Engineering and Applied Sciences, Harvard University, Cambridge, MA 02138

${ }^{2}$ School of Aerospace, Xi'an Jiaotong University, Xi'an, 710049, P.R. China

\begin{abstract}
Cylindrical actuators are made with dielectric elastomer sheets stiffened with fibers in the hoop direction. When a voltage is applied through the thickness of the sheets, large actuation strains are achievable in the axial direction, with or without pre-straining and mechanical loading. For example, actuation strains of $35.8 \%$ for a cylinder with a prestrain of $40 \%$, and 28.6\% for a cylinder without pre-strain have been achieved without any optimization. Furthermore, the actuation strain is independent of the aspect ratio of the cylinder, so that both large strains and large displacements are readily actuated by using long cylinders.
\end{abstract}

Corresponding authors:

a) David R. Clarke, clarke@seas.harvard.edu

b) Zhigang Suo, $\underline{\text { suo@seas.harvard.edu }}$ 
Electroactive polymer (EAP) actuators have been widely studied as artificial muscles for diverse applications, including robotics, ${ }^{1-3}$ motors, 4 adaptive optics, ${ }^{5-7}$ Braille displays, ${ }^{8}$ and bioengineering..$^{9-11}$ Within the family of EAP actuators, such as those made of polymer gels, ${ }^{12}$ ionic polymer-metal composites, ${ }^{13}$ conjugated polymers, ${ }^{14}$ carbon nanotubes, ${ }^{15}$ electrostrictive polymers ${ }^{16}$ and liquid crystal elastomers, ${ }^{17}$ dielectric elastomer actuators are considered particularly attractive because they resemble natural muscles, producing fast and large deformation in response to applied voltage. ${ }^{18}$

Achieving large voltage-induced deformation, however, is a practical challenge. When a voltage is applied across the thickness of an elastomer sheet, the sheet reduces its thickness and expands its area. For a stiff elastomer, an applied voltage causes electric breakdown while the deformation is small. For a soft elastomer, an applied voltage may induce a "pull-in" instability such that the elastomer thins down drastically, leading to electric breakdown rather than actuation.19-23 The instability is greatly affected by boundary conditions, so that the achievable voltage-induced deformation strongly depends on how the mechanical load are applied. While large voltage-induced deformation has been demonstrated for an elastomer sheet under equal-biaxial forces, ${ }^{24-26}$ only small voltage-induced deformation has been observed for an elastomer sheet under a uniaxial force. ${ }^{27}$ Many applications, however, require muscle-like actuators in which a voltage induces large and unidirectional displacement.

In this contribution, we demonstrate that large, unidirectional, voltage-induced displacements can be achieved by using stiff, parallel fibers to constrain the elastomer sheet in the other direction. Our recent work has considered flat fiber-stiffened elastomer sheets, ${ }^{27}$ here we demonstrate them in cylindrical actuators, a configuration that is readily constructed and suitable for a variety of applications. We show that large uniaxial actuation strains can be produced, with or without pre-strains and mechanical loads. We further show that, while large voltage-induced strains are achievable for 
short cylinders made of elastomer sheets without fibers, large actuation strains can be achieved with cylinders of any length made of fiber-stiffened elastomer sheets. Consequently, cylinders of fiber-stiffened elastomer sheets can be readily actuated to create both large strains and large displacements.

For comparison, we present results for the actuation of cylinders without fiber stiffening (Fig. 1). For a long cylinder subject to an axial force, an actuation strain of $4.5 \%$ has previously been reported. ${ }^{28}$ In our experiments, each actuator consisted of a sheet of an acrylic elastomer (VHB 4910, 3M company), thickness $H=1 \mathrm{~mm}$, with carbon-grease electrodes brushed on both surfaces of the sheet (Fig.1). The sheet was attached to two short and stiff $\mathrm{PVC}$ rings of outside diameter $D=4.5 \mathrm{~cm}$. Denote $L$ as the length of the cylinder in the undeformed state. Because of the visco-eleastic behavior of the acrylic elastomer, the cylinder was allowed to creep first under a constant force $P$ for 30 minutes. This caused the length of the cylinder to increase from $L$ to $l_{\text {pre }}$, giving a pre-strain $\xi_{\text {pre }}=\left(l_{\text {pre }} / L\right)-1$. Then a voltage $\Phi$ (Gamma High voltage Research, Model NO.: ES40P-5W-DAM) was applied at a fixed ramp rate of $50 \mathrm{~V} / \mathrm{s}$, while digital images of the deforming cylinder were recorded. Subject to both the force $P$ and voltage $\Phi$, the cylinder elongated from $l_{\text {pre }}$ to $l$, giving an actuation strain $\xi_{\text {act }}=\left(l / l_{\text {pre }}\right)-1$.

This experiment was repeated for cylinders of three different lengths, $L=$ 1.0, 4.0, and $10.0 \mathrm{~cm}$, and a constant force $P=767 \mathrm{~g}$. The strains produced in the three cylinders by the constant force after 30 minutes before applying the voltage were $\xi_{\text {pre }}=113 \% 118 \%$ and $120 \%$, respectively. The actuation strains increased as the voltage was ramped up as shown in Fig. 1 until electric breakdown occurred. The data showed that the achievable actuation strain was smaller the longer the cylinder (Fig.1d). The actuation strain achieved was $\xi_{\text {act }}=37.3 \%$ for the cylinder of length $L=1 \mathrm{~cm}$, but was only $\xi_{\text {act }}=14.3 \%$ for the cylinder of length $L=10 \mathrm{~cm}$. Inspection of the images recorded 
concurrently provided a qualitative explanation for this behavior. When the force $P$ was applied, the elastomer stretched in the axial direction but also narrowed in the radial direction, forming a waist (Fig. 1b). The diameter was narrowest in the centre and widens to match that of the rigid rings at either end indicating that the rings constrained the deformation of the elastomer in their immediate vicinity. When the voltage was applied the cylinder not only increased in length axially but also expanded radially, offsetting the waist produced by the mechanical force alone. For the shorter cylinders, $L=1.0$ and $4.0 \mathrm{~cm}$, the cylinders could recover the initial central radius under the action of the applied voltages, and even formed wrinkles before electric breakdown (Fig. 1c). For the longest cylinder, $L=10.0$, however, electric breakdown took place when the actuation strain was relatively small, and the waist was still apparent.

We attribute the large axial actuation strains achieved by the short cylinders to the constraint afforded by the rigid PVC rings at their ends. The situation is similar to a flat elastomer sheet constrained at the top and bottom edges by two rigid clamps. When the sheet is long in the horizontal direction and short in the vertical direction, the rigid clamps constrain the deformation in the horizontal direction, and large actuation strains have been observed in the vertical direction. ${ }^{29}$ By contrast, for the longer cylinder, the elastomer away from the PVC rings is unconstrained in the radial direction, and is in effect under only a uniaxial force. For an elastomer sheet under a uniaxial force, the larger the applied force, the smaller the achievable voltage-induced strain. ${ }^{27}$ Although the large axial strains achieved with the short cylinders of unstiffened elastomers may be useful, large displacements $\left(l-l_{p r e}\right)$ cannot be achieved this way, unless multiple sections of elastomer cylinders, each separated and constrained by a rigid ring, are connected in series. While this serial configuration may be useful for various forms of articulation, the rigid rings would add considerable mass to the actuator.

These considerations motivated us to explore using parallel fibers to 
stiffen the elastomer in the hoop direction so as to maximize actuation strains in the axial direction. The fiber stiffened acrylic was produced by hand, laying up $0.2 \mathrm{~mm}$ diameter nylon fibers, spaced $2 \mathrm{~mm}$ apart and parallel to one another (Fig. 2). A second sheet of acrylic was pressed down on top of the first sheet, so that the fibers were sandwiched between the two sheets. The fiber-stiffened double sheet was then coated with carbon on both sides, and rolled into a cylinder with the PVC rings at the two ends.

As might be expected, under mechanical load a cylinder of fiber-stiffened elastomer resisted radial deformation and did not form a noticeable waist (Fig. 3). Furthermore, the actuation strain was independent of the length of the cylinder: the data for the cylinders of lengths $L=10 \mathrm{~cm}$ and $4 \mathrm{~cm}$ superimposed. These observations indicate that the closely-spaced fibers prevent elongation in the hoop direction, and the cylinder deforms in the axial direction. Because the actuation strain is independent of the length of the cylinder, both large unidirectional actuation strains and actuation displacements can be readily achieved using long cylinders.

As the actuation strain was independent of the length of the cylinder, for the experimental convenience, we used the short cylinders $(L=4 \mathrm{~cm})$ to study the effect of a superimposed mechanical force. Four different mechanical loads, $P=0,547,767$ and $877 \mathrm{~g}$, were used, resulting in pre-strains $\xi_{\text {pre }}=0$, $40 \%, 80 \%$, and $108 \%$, respectively. All four actuators worked well, from the largest actuation strain of $35.8 \%$ under a pre-strain of $\xi_{\text {pre }}=0.4$, to the smallest actuation strain of $28.6 \%$ under no pre-strain (Fig. $4 \mathrm{a}$ ). When the applied pre-strain was too large, however, the fibers buckled, and the actuator would gradually lose the advantages of using a fiber-stiffened elastomer (Fig. $4 b)$.

Our finding that a large axial strain can be achieved even without any mechanical pre-strain is of practical importance to the development of dielectric elastomer actuators. It also considerably simplifies the fabrication of 
dielectric actuators since there is no need to incorporate structural elements and designs for creating and maintaining large pre-strains in the elastomer material. In addition, it is also anticipated that larger actuation strains will be achievable once the fiber spacing, diameter and stiffening have been optimized.

In summary, we have shown that cylindrical actuators made of fiber-stiffened elastomer sheets produce large uniaxial actuation, even without pre-straining. Furthermore, the actuation strains are independent of the aspect ratio of the cylinder, so that both large actuation strains and large actuation displacements are readily achievable using long cylinders. By contrast, without the fiber stiffening, large actuation strains are only achievable when the applied force is small and when the length of the cylinder is small compared with the diameter of the cylinder. Fiber-stiffened dielectric elastomers are an unusual "product" composite material in that the fibers not only amplify the attainable deformation when an electric field is applied but also suppress electrical breakdown in their immediate vicinity. Also, although the emphasis of the current contribution has been on the use of fibers to create local stiffening, it is anticipated that other means of directional stiffening and enhancing actuation can be realized. These might include laminating a dielectric elastomer with a passive material of anisotropic stiffness, as well as producing anisotropic dielectric elastomers through molecular engineering.

The work was supported by DARPA (W911NF-10-1-0113), and by the MRSEC at Harvard under award DMR-080484. Lu is a visiting scholar at Harvard University sponsored by the China Scholarship Council. 


\section{References}

1. R. Pelrine, R. Kornbluh, Q. Pei, S. Stanford, S. Oh, J. Eckerle, R. Full, M. Rosenthal, and K. Meijer, In SPIE Electroactive Polymer Actuators and Devices 4695, p. 126 (2002)

2. G. Kofod, W. Wirges, M. Paajanen, and S. Bauer, Appl. Phys. Lett. 9o, 081916 (2007).

3. G. Kovacs, L. During, S. Michel, G. Terrasi, Sensors Actuators A 155, 299 (2009).

4. I. A. Anderson, T. Hale, T. Gisby, T. Inamura, T. McKay, B. O’Brien, S. Walbran, and E P. Calius, Appl. Phys. A 98, 75 (2010).

5. G. Kofod, D. N. McCarthy, J. Krissler, G. Lang, and G. Jordan, Appl. Phys. Lett. 94, 202901 (2009).

6. M. Beck, R. Fiolka, and A. Stemmer, Optics Lett. 34, 803 (2009).

7. F. Carpi , G. Frediani , S.Turco, and D. De Rossi, Adv. Funct. Mater. 21, 4152 (2011).

8. Z.B. Yu, W. Yuan, P. Brochu, B. Chen, Z.T. Liu, and Q.B. Pei, Appl. Phys. Lett. 95, 192904 (2009).

9. S. Akbari and H.R. Shea, J. Micromech. Microeng. 22, 045020 (2012).

10. V. L. Finkenstadt, Appl. Microbiol. Biotechnol. 67, 735 (2005).

11. N. K. Guimard, N. Gomez, and C. E. Schmidt, Prog. Polym. Sci. 32 , 876 (2007).

12. H. B. Schreyer, N. G., K. J. Kim, and M. Shahinpoor, Biomacromolecules 1, 642 (2000)

13. J. D. W. Madden, N. A. Vandesteeg, P. A. Anquetil, P. G. A. Madden, A. Takshi, R. Z. Pytel, S. R. Lafontaine, P. A. Wieringa, and I. W. Hunter, IEEE J. Ocean. Eng. 29, 706 (2004).

14. E. W. H. Jager, E. Smela, and O. Ingana, Science 290, 1540 (2000).

15. R.H. Baughman, C. Cui, A. A. Zakhidov, Z. Iqbal, J. N. Barisci, G.M. Spinks, G. G. Wallace, A. Mazzoldi, D. D. Rossi, A. G. Rinzler, O. Jaschinski, S.

Roth, and M. Kertesz, Science 284, 1340 (1999).

16. Q. M. Zhang, V. Bharti, and X. Zhao, Science 280, 2101 (1998).

17. W. Lehmann, H. Skupin, C. Tolksdorf, E. Gebhard, R. Zentel, P. KruÈ ger,

M. LoÈsche, and F. Kremer, Nature 410, 447 (2001).

18. P. Brochu and Q. Pei, Macromol. Rapid Commun. 31, 10 (2010).

19. R.E. Pelrine, R.D. Kornbluh, and J.P. Joseph. Sensors and Actuators A 64, 77 (1998).

20.M. Wissler and E. Mazza, Sensors and Actuators A 120, 184 (2005).

21. J.-S. Plante and S. Dubowsky, Int. J. Solids Struc. 43, 7727 (2006). 
22. E.M. Mockensturm and N. Goulbourne, Int. J. Non-Linear Mech. 41, 388 (2006).

23.X.H. Zhao and Z.G. Suo, Phys. Rev. Lett. 104, 178302 (2010).

24. R. Pelrine, R. Kornbluh, Q. Pei., and J. Joseph, Science 287, 836 (2000).

25. S. J. A. Koh, T.F. Li, J.X. Zhou, X.H. Zhao, W. Hong, J. Zhu, and Z.G. Suo, J. Polym. Sci., Part B: Polym. Phys. 49, 504 (2011).

26.J. Huang, T. Li, C. C. Foo, J. Zhu, D. R. Clarke, and Z.G. Suo, Appl. Phys. Lett. 100, 041911 (2012).

27. T.Q. Lu, J.S. Huang, C. Jordi, G. Kovacs, R. Huang, D.R. Clarke, and Z.G. Suo, manuscript submitted for publication.

28. F. Carpi and D. De Rossi, Mater. Sci. Eng. C 24, 555 (2004).

29. M. Kollosche, J. Zhu, Z.G. Suo, and G. Kofod, manuscript Submitted for publication. 
(a)

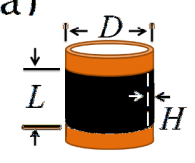

(b)

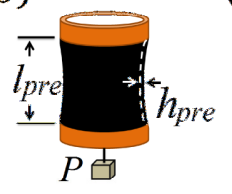

(c)
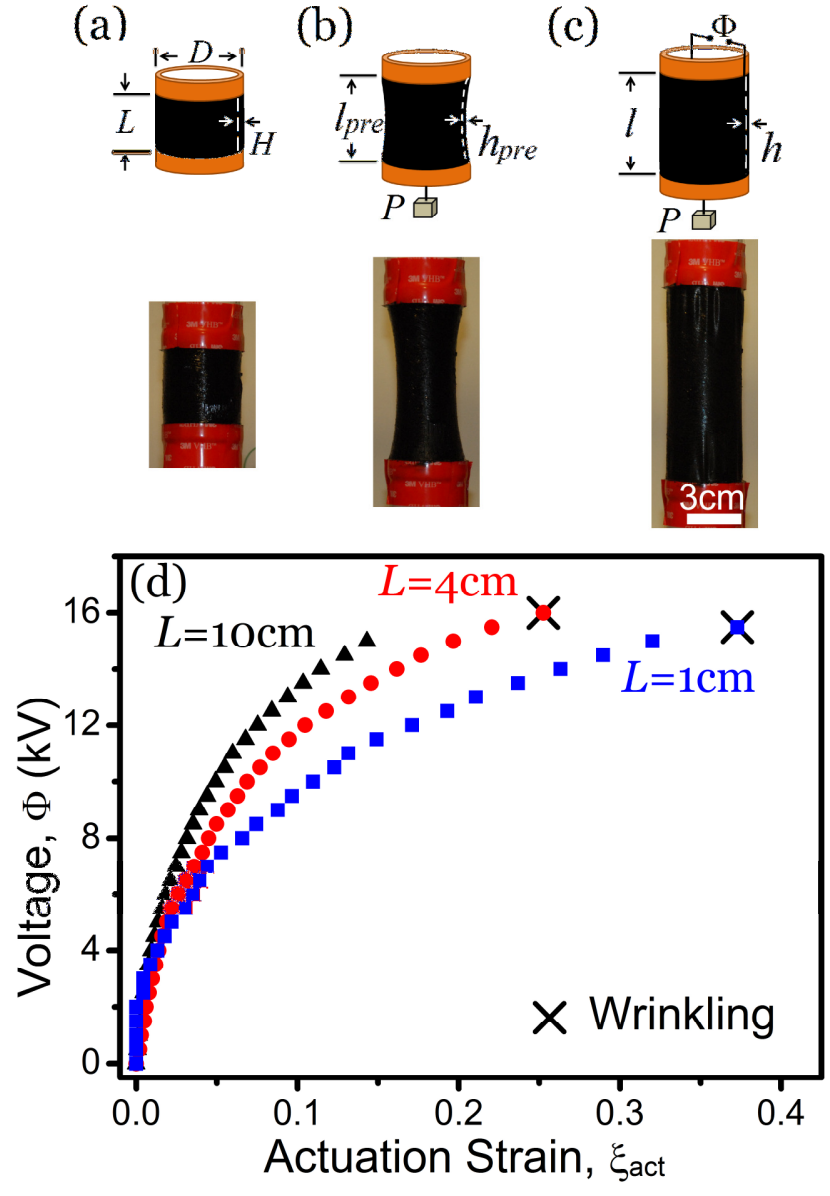

FIG. 1. Response of cylindrical actuators made of elastomer sheets without fibers. (a) In the reference state, the elastomer is undeformed. (The elastomer is black because of the carbon electrode coating). (b) The cylinder is in a pre-stretched state due to an applied force $P$. Note the formation of a waist in the central region of the actuator. (c) In the actuated state, the membrane is subject to both the force $P$ and voltage $\Phi$. The red ends are stiff rings on which the elastomer is attached. The three states are illustrated with both schematics and photographs of a cylinder of length $L=4 \mathrm{~cm}$. Cylinders of length $L=1 \mathrm{~cm}$ and $4 \mathrm{~cm}$ formed wrinkles before electric breakdown. (d) For cylinders of different lengths, the actuation strains were recorded as the voltage was ramped up. The successive data points were recorded at equal time intervals of 10s. Each run of the experiment was terminated at electric breakdown. 


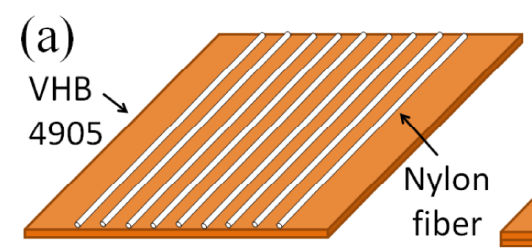

(b)
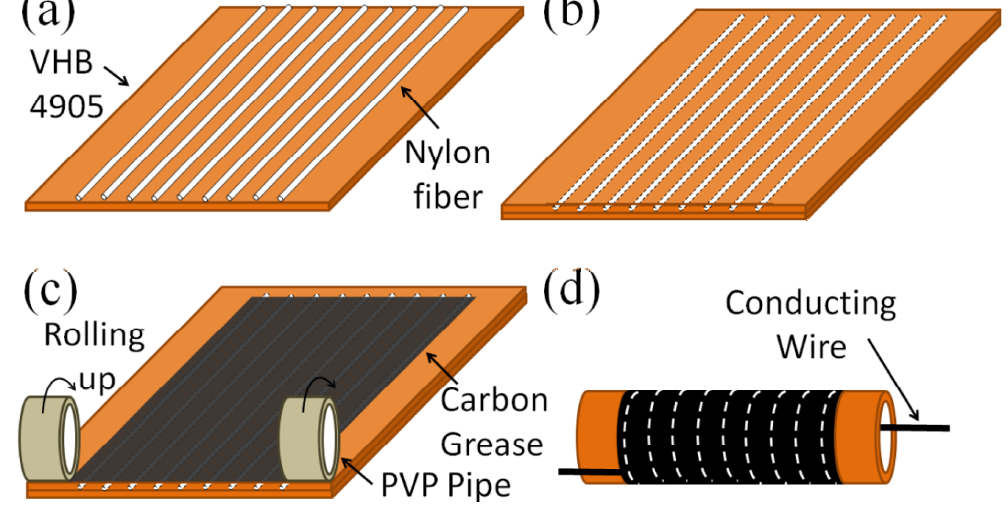

FIG. 2. Schematic illustration of the process used to make fiber-stiffened elastomer sheets and a cylindrical actuator . (a) Nylon fibers of diameter 0.2 $\mathrm{mm}$ were placed parallel to one another and $2 \mathrm{~mm}$ apart on the surface of a sheet of VHB 4905 - (b) A second sheet of VHB 4905 was placed on the top and then compressed to bond them together. (c) One surface of the combined sheet was brushed with carbon grease, and rolled onto two short PVP rings of outside diameter $4.5 \mathrm{~cm}$ at each end. (d) Carbon grease was also brushed on the outside surface of the cylinder, and conducting wires attached to the carbon-grease electrodes inside and outside of the cylinder to form the complete actuator. 
(a)

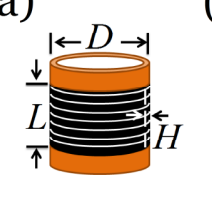

(b)

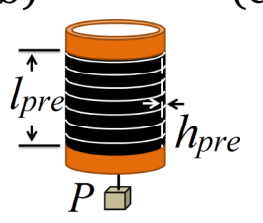

(c)

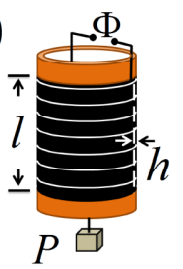

3cm

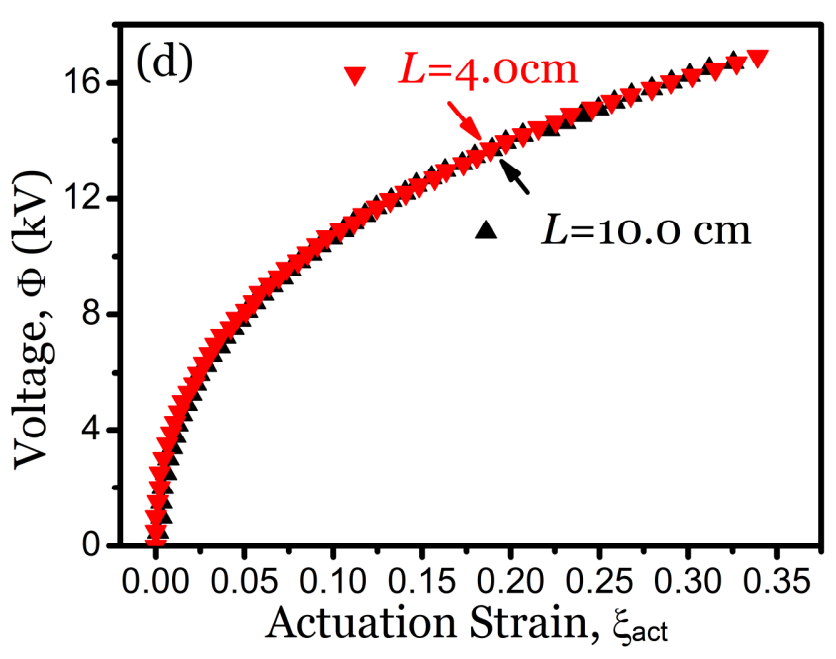

FIG. 3. Actuation of cylinders made of fiber-stiffened elastomer sheets. (a, b, c) Schematics and the photographs of fiber-stiffened cylindrical actuator in the reference state, the prestretched state, and the actuated state. (d) Under a constant force of $P=767 \mathrm{~g}$ and a prestrain of $80 \%$, the electric actuation strains were measured for cylinders of two different lengths $L=4 \mathrm{~cm}$ and 10 $\mathrm{cm}$. 

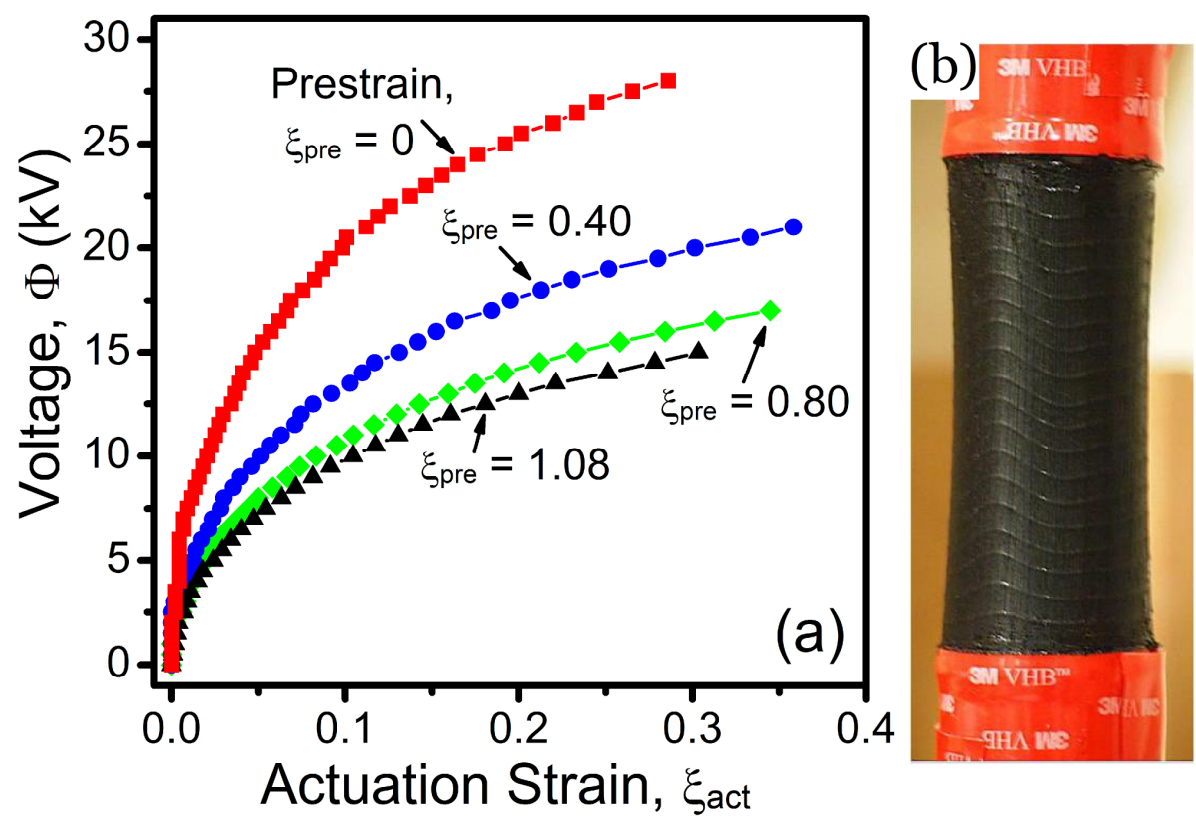

FIG. 4. (a) The voltage induced actuation strains for four cylinders with different values of pre-strain, including one not pre-strained. In each case, the maximum strain was limited by electric breakdown. (b) The fibers buckled when the prestrain is too large. The photograph shows the buckling pattern in a cylinder at a pre-strain of $155 \%$. 